\title{
Active materials for adaptive architectural envelopes based on plant adaptation principles
}

\author{
Marlén López ${ }^{\mathrm{a}, *}$, Ramón Rubio ${ }^{\mathrm{a}}$, Santiago Martín ${ }^{\mathrm{a}}$, Ben Croxford ${ }^{\mathrm{b}}$ and Richard Jackson ${ }^{\mathrm{c}}$ \\ ${ }^{a}$ Department of Construction and Manufacturing Engineering. University of Oviedo, IDEASCAD \\ Research Group. C/ Pedro Puig Adam. Campus de Viesques, Gijon, Spain \\ ${ }^{\mathrm{b}}$ The Bartlett, Faculty of the Built Environment. UCL \\ 'Institute of Biomedical Engineering. UCL
}

\begin{abstract}
In this paper, the authors present research into adaptive architectural envelopes that adapt to environmental changes using active materials, as a result of application of biomimetic principles from plants to architecture. Buildings use large amounts of energy in order to maintain their internal comfort, because conventional buildings are designed to provide a static design solution. Most of the current solutions for facades are not designed for optimum adaptation to contextual issues and needs, while biological solutions to adaptation are often complex, multi-functional and highly responsive. We focus on plant adaptations to the environment, as, due to their immobility, they have developed special means of protection against weather changing conditions. Furthermore, recent developments in new technologies are allowing the possibility to transfer these plant adaptation strategies to technical implementation. These technologies include: multi-material 3D printing, advances in materials science and new capabilities in simulation software. Unlike traditional mechanical activation used for dynamic systems in kinetic facades, adaptive architectural envelopes require no complex electronics, sensors, or actuators. The paper proposes a research of the relationship that can be developed between active materials and environmental issues in order to propose innovative and low-tech design strategies to achieve living envelopes according to plant adaptation principles.
\end{abstract}

Keywords: Active materials, adaptive architecture, biomimetics, energy efficiency, plants

\section{Introduction}

"Cities are part of the climate change problem, but they are also a key part of the solution." (KamalChaoui \& Robert, 2009). Nowadays cities consume the larger part of global energy and are therefore major contributors of greenhouse gas emissions. Moreover, cities have key competencies to act on climate change through their responsibilities over urban sectors such as buildings. So much so that latterly the European Union has been developing a large number of funding building efficiency programs for research and innovation trying to problem-solve these issues, such as the Horizon 2020 framework. Some of these programs focus on building retrofitting, or the installation of energy-efficient

\footnotetext{
*Corresponding author: Marlén López, Department of Construction and Manufacturing Engineering. University of Oviedo, IDEASCAD Research Group. C/ Pedro Puig Adam. Room 6.1.19. Campus de Viesques, 33203 Gijon, Spain. Tel.: +34 656 181913; E-mail: arqmarlenlopez@gmail.com.
} 
technologies, especially on facades. Facades have an important role in the regulation and control of energy waste, since they act as intermediary filters between external environmental conditions and inside users and functional requirements. Due to this decisive role, in recent years facades or architectural envelopes have been the subject of numerous studies and research, always trying to achieve greater efficiency and performance in terms of energy, comfort or structure (Almusaed, 2011; Fortmeyer \& Linn, 2014; Park \& Dave, 2014).

As opposed to our buildings, which remain inert, living objects respond to the environment and they are able to adapt to the changing weather conditions (Armstrong, 2012). Innovation in technological functions of architecture is key to meeting these challenges. It should be noted that the emergence of new construction techniques, such as additive manufacturing, allows to break the geometric constraints of traditional processes (Spiller \& Armstrong, 2011).

This research is about an architecture that continuously changes in response to external stimuli. Through technological innovation we investigate the application of biomimetics to the development of adaptive architectural envelopes according to plant adaptation strategies. Advances in technology have opened new avenues for design; we can more effectively mimic nature's language (Oxman, 2012).

This paper is organized in two main sections. In the first one, motivations for application to biomimetic principles from plants to architecture are explained, as well as concepts such as adaptation and why plant adaptation principles are the basis of this research. The second section is related to how to convert these inspirational mechanisms of plants into technical implementations for adaptive architectural envelopes. A selection of possible active materials is proposed, according to the environmental issues defined: humidity, temperature, carbon dioxide and light. Finally some ideas about possible manufacturing tools are described.

\section{From plant adaptations to architecture}

\subsection{Adaptation}

Adaptation is the evolutionary process whereby an organism becomes better able to live in its habitat or habitats (Dobzhansky et al., 1968).

The multiple environmental and climatic characteristics of the area are variable parameters, while those concerning internal comfort in buildings are largely static; so, we use large amounts of energy to pump heating, or cool, ventilate and light our buildings between quite well defined limits, while external environmental factors can change considerably. The existing solutions to these problems tend to have a static building envelope and dynamic building services. Therefore conventional solutions for facades and roofs are not designed for optimum adaptation to contextual issues and needs. However, biological solutions to adaptation are often complex, multi-functional and highly responsive.

The building envelope, without distinction between walls and roof, is the interface between exterior environmental factors and the interior demands of the occupants (Del Grosso \& Basso, 2010). An adaptive architectural envelope is one that responds to changing environmental conditions both interior and exterior while managing the indoor environment. Adaptive architectural envelopes should have adaptation strategies to anticipate exterior environmental variations as well as interior activities and their interactions with inhabitants. The building envelope could be constructed, not with the traditional inert surfaces, but with a 'living' cladding, which could house a wide range of technologies based on the behaviour of the envelopes found in nature (Beesley, 2006). 
Nowadays biology is a new inspiration for technological thinking. Some of these studies have looked at nature as a source of inspiration for subsequent application to architecture, a trend also known as biomimicry or biomimetics. These terms come from the Greek words bios, meaning life, and mimesis, meaning to imitate. Biomimetics is defined as the 'abstraction of good design from nature' (Vincent, 2006) or 'mimicking the functional basis of biological forms, processes and systems to produce sustainable solutions' (Pawlyn, 2011). Systems found in nature offer a large database of strategies and mechanisms that can be implemented in biomimetic designs. Usually it is a discipline that has been developing for some time in other fields, such as engineering or medicine, however in recent years we begin to see how several research works have been developed around biomimicry to look into new solutions in architecture. Some of them try different methodologies for developing new building envelopes based on biomimetic principles, such as "Towards the living envelope" (Badarnah, 2012) or "Architecture follows nature" (Mazzoleni, 2013). Unlike these studies, this research is based only on plants and their strategies of adaptation to different climates. We only focus on those adaptations to environment shown by plants.

\subsection{Plant adaptation principles}

Plants, like buildings, lack movement and remain subject to a specific location, so they have to resist weather conditions that affect them at all times and they have developed special means of protection against excessive wind, drought, cold, heat and light. Plants, unlike buildings, have adapted to the environment, through processes of evolution over millions of years. These adaptations develop over time and generations as a response to the ever-changing environment. The study of concepts such as Bioclimatology or Phytosociology (Azcón-Bieto \& Talón, 2000) is part of the research process in which plant communities, and their relations with the environment, and the temporal processes that modify them, are analysed. The basis of the success of plants is the ability to compete in their environment and it depends mainly on their physiological evolution and adaptation to the environment. One of the most important applications of physiological principles refers to the study of the relationships between plants and climate, with physical and physiological environmental factors and the relationships between them. Climatic factors include intensity and periodicity of heat and light, precipitation and relative humidity, as well as wind or the periodicity and duration of the seasons. This leads us to the analysis of the distribution of plants in different geographical territories as climatic factors, or, in other words, to Bioclimatology. This information is particularly relevant as an introductory element to the biological analysis as well as the architectural proposition. Taking as a reference the Worldwide Bioclimatic Classification System (Rivas-Martínez, 2004), we focus on Europe, where we can see four of the five broad climate types defined: Mediterranean, Temperate, Boreal and Polar.

In a first biological approach we can validate that evolution and adaptation of plants, like all the organisms, occur in three main ways: morphological, physiological and behavioural. In addition to this first general approach, we define a second more useful one to order the wide range of plant adaptation examples that can be found in nature, as well as make easier the application or transfer solutions from nature to architectural solutions. We establish a classification of plant adaptations and interactions with their environment, so the core of this proposal is movement, resulting in two categories: dynamic mechanisms and static strategies. At the same time, these two main approaches could be further divided into a macro and micro level. Within dynamic mechanisms we consider those plants that respond to external stimuli through movement. We focus on those plants that 

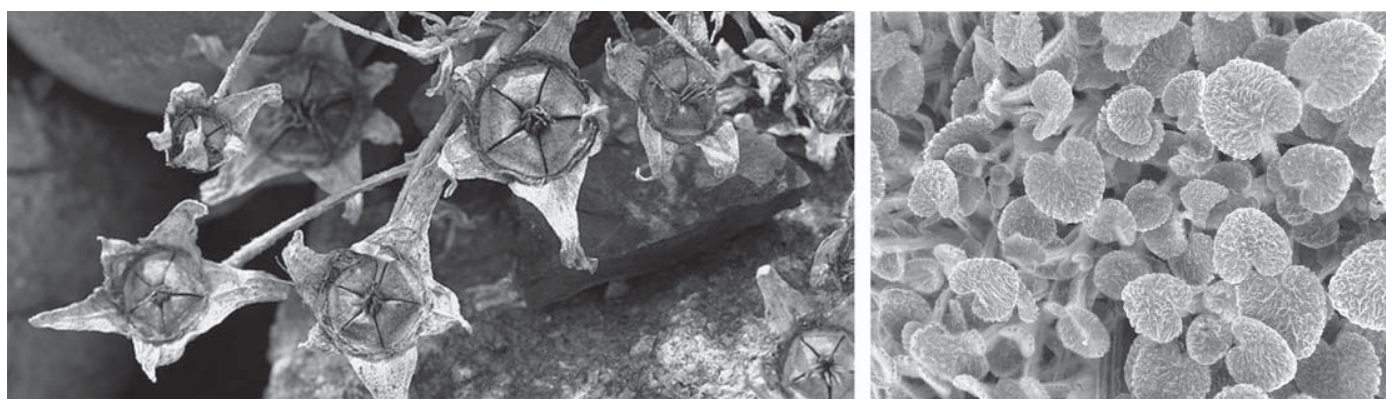

Fig. 1. The image on the left shows a dynamic valve mechanism in seeds of many Mesembryanthemums as a behavioural adaptation, using rainwater as a trigger to open and to launch their seeds. The image on the right shows a static strategy of hairy leaves of Gynandriris setifolia as a morphological adaptation to protect themselves against excessive sunlight and temperature, by strategies of three-dimensional waxes or dense coverage with air-filled hairs.

are responsive, that is, exhibit rapid and reactive movements, in a timescale that we can perceive. In this way, we study how plants react in specific ways to light, temperature, fire, darkness, water or drought through reactive mechanisms in the macroscopic and microscopic scales. On the other hand, within static strategies we focus on the highly multifunctional properties of plant leaves. Plant surfaces provide more than one solution for environmental conditions and can include, for example, light reflection, superhydrophobic or superhydrophilic surfaces (Koch et al., 2009; Bhushan, 2009; Gibson, 2012). In Fig. 1 we can see how plants from the Mediterranean climate (dry and hot areas) present different adaptations, with dynamic mechanisms or static strategy depending on the case.

This study is part of a larger research and we have found stomata of leaves of particular interest once several plant adaptations examples have been studied for their possible application to adaptive architectural envelopes. We will see in the following section how stomata are an example of dynamic mechanisms and, at the same time, static strategies, and they demonstrate that the classification proposed is not exclusive and therefore stomata are specimens with an exceptional value in the process of biomimetic inspiration.

\subsection{Stoma}

Definition of stoma, or stomata in plural, related to botany, is that it is a pore, found in the epidermis of leaves used to control gas exchange. The pore is bordered by a pair of specialized parenchyma cells known as guard cells that are responsible for regulating the size of the opening. Guard cells perceive and process environmental and endogenous stimuli such as light, humidity, carbon dioxide, temperature, drought, and plant hormones to trigger cellular responses resulting in stomatal opening or closure (Azcón-Bieto, J. \& Talón, M., 2000; Vogel, 2012)

As explained above, stomata are chosen between all possible adaptations in plants because they are an example of dynamic mechanisms and static strategies (Fig. 2). Stomatal valve movements in response to water and carbon dioxide interchanges are an example of dynamic mechanisms. In addition, there is a great variability in morphologies on surface structures around these valve cells, due to functional adaptations to environmental conditions. Also, these modifications of surfaces are examples of static mechanisms. Stomata are responsible for the interchange of gases for respiration and photosynthesis. Furthermore, stomata allow for the loss of excess water in the form of water vapour, which also allows for cooling. So, functions 

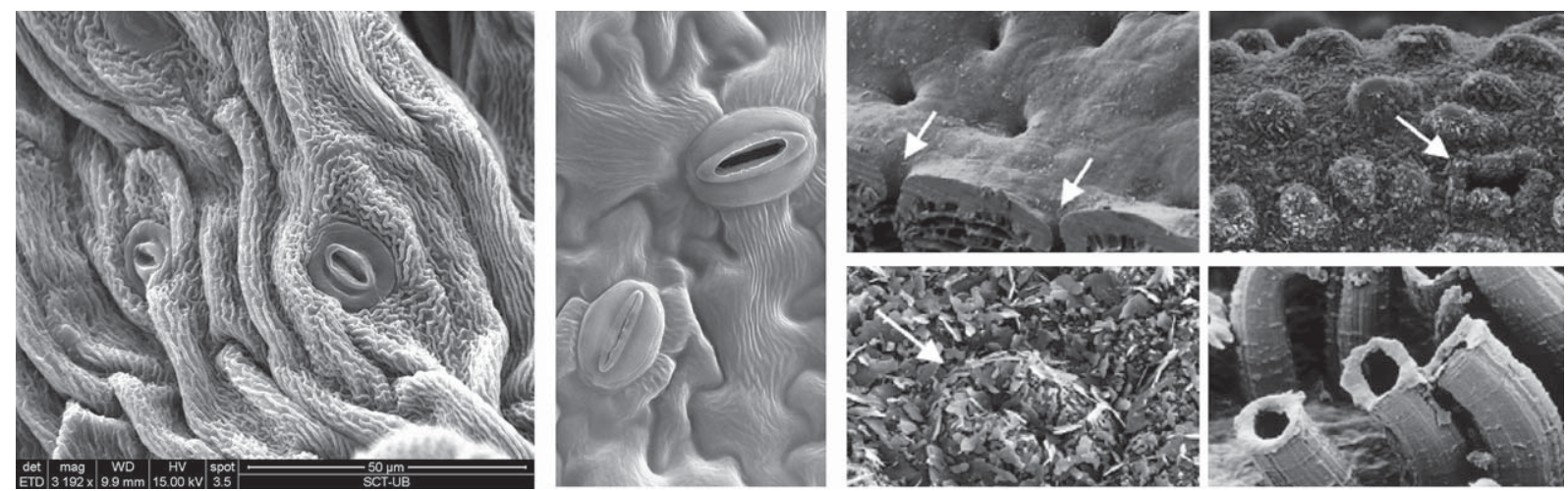

Fig. 2. From left to right: stomata on Crucifera leaves; stomatal valve movements on a Lavandula dentata leaf; different morphologies of sunken stomata, waxes, hair structure or chimneys to reduce the evaporation of water.

\begin{tabular}{|c|c|c|c|}
\hline ENVIRONMENTAL ISSUES & CONTROLLED VARIABLE & & STOMATAL MOVEMENT \\
\hline $\begin{array}{l}\text { humidity or } \\
\text { water availability } \\
\text { temperature }\end{array}$ & $\begin{array}{l}\mathrm{CO}_{2} \text { concentration } \\
\text { within a leaf }\end{array}$ & $\begin{array}{l}\text { low concentration } \\
\text { high concentration }\end{array}$ & $\begin{array}{l}\text { OPENING } \\
\text { CLOSURE }\end{array}$ \\
\hline $\begin{array}{l}\text { atmospheric carbon } \\
\text { dioxide concentration } \\
\text { light intensity }\end{array}$ & $\begin{array}{c}\mathrm{H}_{2} \mathrm{O} \text { level (turgidity) } \\
\text { within a leaf }\end{array}$ & $\begin{array}{l}\text { high level } \\
\text { low level }\end{array}$ & $\begin{array}{l}\text { OPENING } \\
\text { CLOSURE }\end{array}$ \\
\hline
\end{tabular}

Fig. 3. Diagram showing the mechanism of stomatal movement.

of stomata include: interchanging of gases, avoiding lack of water, transpiration and interchanging of temperature. A leaf must continuously adjust the apertures of its stomata as conditions (light intensity and water and dioxide carbon availability) change. Stomata open in response to a decrease in concentration of dioxide carbon, as well as respond directly to light. Temperature provides another stimulus: at higher temperatures, stomata commonly open, responding to increased carbon dioxide consumption and they close responding to the higher level of carbon dioxide. Finally stomata respond to water or high humidity through guard cells that increase their turgidity by opening (Vogel, 2012; Martin et al., 1983). Thus, control of stomatal movements depends on the controlled variable within the leaf and the external inputs (Fig. 3). Controlled variables are dioxide concentration and the water level (turgidity). External inputs are humidity or water availability, temperature, atmospheric carbon dioxide concentration and light intensity.

Besides this mechanism of stomatal movement, different surface structures have been developed in leaves around stomata to adapt to environmental conditions. We can find different wax morphologies and hair structures, on plants from the Temperate climate, whose role is to reduce the evaporation of water. Sunken stomata, wax chimneys around the stomata or wax-covered stomata, are found out on plants from the Mediterranean climate, in dry and hot habitats. In the same way dense coverage with air-filled hairs, is used to reflect visible light and temperature control, as well as to absorb 
UV-radiation. We also consider other features in their anatomy such as size and frequency or density (number of stomata) (i.e: in arid zones plants show a reduction per leaf area to reduce water loss) (Koch et al., 2009). Therefore, we have the key of their environmental adaptations and interactions by studying the micro-structures of the stomata of plants at different climate zones. It is important to understand the principles of adaptation solutions and transferring them into artificial systems for adaptive architectural envelopes rather than simply copying them.

As part of a large research, transfer from stomata challenges into architectural design objectives will be carried out through a design concept generation methodology. This methodology has been created to guide the design process from biological challenge, its function and main features, to some innovative ideas according design challenges and benefits, as well as to technical implementation with its features. We are in a preliminary design phase from stomata to technical implementation, and some results of this transfer have not been achieved yet. What follows is a short definition of functions for the architectural envelope, as an adaptive interface between environmental issues and internal comfort. Identification of these functions will lead us to define innovation challenges in adaptive architectural envelopes compared to a standard or traditional building system in terms of energy efficiency.

\section{Environmental issues and active materials}

\subsection{Environmental issues}

The environment is constantly changing and producing new challenges to cope with. Light (solar radiation), temperature, relative humidity, rainwater, wind (air movement), noises and carbon dioxide (air quality) are the basic environmental issues affecting a building. These issues significantly affect occupant comfort demands as well as building performance. We have seen how stomatal movements and different microstructures have a great influence as interfaces between plant and environmental issues. Humidity, temperature, carbon dioxide and light are the external inputs that have an effect on stomatal movements. Based on this lesson from nature, the objective of this research is to transfer this biological mechanism into a new system for architectural envelopes capable of adapting to the same issues in order to be more efficient and less energy demanding. Therefore humidity, temperature, carbon dioxide and light, are the environmental issues selected in this research and others such as rainwater, air movement or noises are not taken into account.

External inputs depend directly on the specific climatic zone - data given by the Worldwide Bioclimatic Classification System - as well as the season and the daytime or night-time period. For user demands inside the building some standard regulations for buildings are available, such as ASHRAE (American Society of Heating, Refrigerating, and Air-Conditioning Engineers) and CTE (Código Técnico Edificación), according to the level and type of activity. Based on those demands, the following functions are defined for the architectural envelope, as an adaptive interface between environmental issues and internal comfort:

- Absorbing, collecting or evaporating to regulate humidity

- Dissipating, gaining or conserving to regulate temperature

- Filtering or exchanging to regulate carbon dioxide (air quality)

- Reflecting, absorbing, redirecting or diffusing to regulate light 


\subsection{Active materials}

After defining biological inspiration how can we materialize this mechanism into a technical implementation? This research tries to work out a system capable of growing or shrinking in size or changing in shape to adapt to different environmental issues, through the ingrained properties of the material they are made of, without the need for external energy or complex mechanical parts. We study the possibilities of fabrication of responsive systems, where multiple materials can react to the environment and deform over time, such as mechanisms that transform into a predetermined shape, changing property and function after fabrication through deformation of active materials capable to stretching, folding or bending, depending on environmental stimulus. However, traditional materials such as ceramics, metals or glasses are industrially produced to satisfy the demands of the building sector, so they are homogeneous and uniform in composition, and isotropic, having identical or very similar properties in all directions (Menges, 2012). Thus, conventional materials and manufacturing processes only provide inert solutions, static results or complex high-tech equipment to achieve kinematic systems. Usually, kinetic architecture is developed through mechanical and electronic sensing, actuating and regulating devices, resulting in a non energy-efficient architecture. It seems clear that it would be very difficult to achieve our purpose by means of inert traditional materials.

On the other hand, a wide range of smart materials has emerged in recent years. Smart materials have properties that react to changes in their environment. The main research directions are based on shape memory alloys (SMA), shape memory polymers (SMP), piezoelectric materials, magnetostrictive materials, electrostrictive materials and electroactive polymers. There are several ways to classify different types of smart materials. We can mention the one carried out by Addington (Addington \& Schodek, 2004) where two kinds of smart materials are described: property change materials, that changes one of their properties (chemical, mechanical, optical, electrical, magnetic or thermal) in response to a change in the conditions of their environment, and do so without the need of external control and energy exchange materials that transform energy from one form to another to effect a desired final state. In addition, in some material centres, information related to smart materials is organized directly according properties and features, such as photoluminescence, electrochromism, shape memory, phase change or magnetorheology among others. In summary, most common active materials are those relying on electrical stimulus to activate movement, such as piezoelectric ceramics. However, this kind of smart materials has been dismissed for our selection, because we focus on low-tech and low-energy adaptive material systems, rather than highly automated and mechanical systems. We are interested in those materials that have structural and physical properties to generate movement or kinetically adapt in real time to environmental changes; active materials, with kinematic behaviours for a better performance that shrink, fold or expand responding to changes and, at the same time, remain stable in their different configurations. What follows is a description of a first approach to active materials with high potential to reduce energy consumption according to the environmental issues explained in previous sections.

We look into self-actuating responsive materials with innate characteristics, behaviour and performative capacity to react to humidity, temperature, carbon dioxide and light. These atmospheric conditions act as 'green' triggers on active materials with reversible changes.

\subsubsection{Humidity reactive materials}

Hydrogel: smart gel based on an insoluble network of polymer chains that swell up when water is added, making an expanded mass. Hydrogels are highly absorbent, they can store large amounts of 


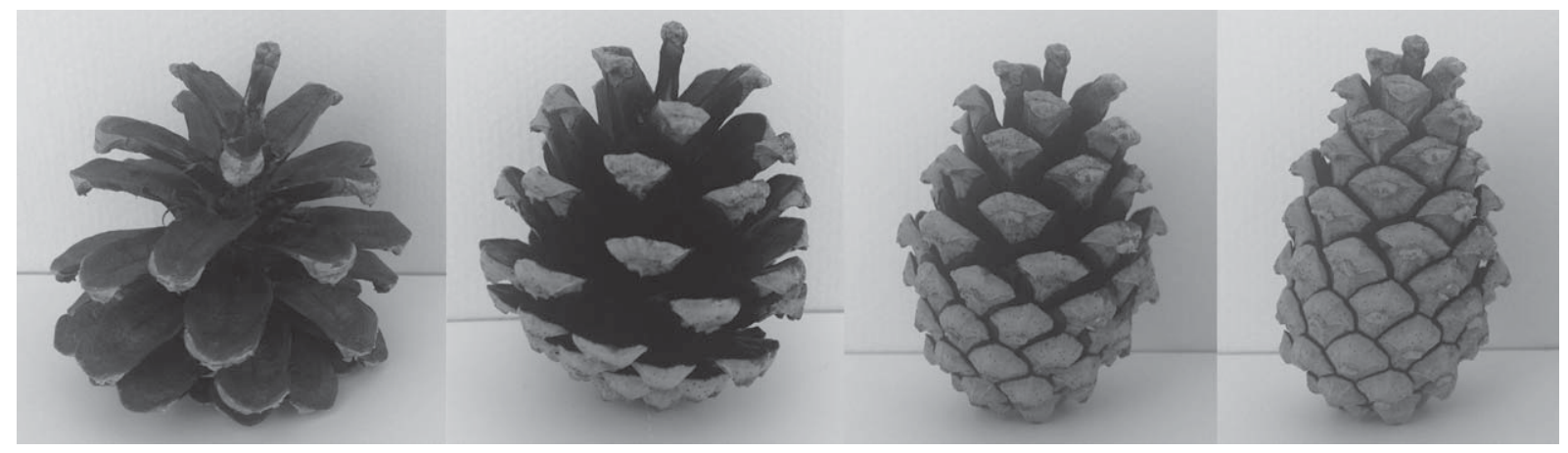

Fig. 4. Experiments of opening and closing movements in conifer cones due to humidity level changes.

water, they also possess a degree of flexibility very similar to natural tissue, due to their significant water content.

Wood: wood is a traditional material, however we include it in this list due to its properties of hygroscopicity and anisotropy, which convert wood into a humidity reactive material. Wood is a cellular structure and always seeks to reach equilibrium moisture, thus continuously responding to changes in the relative humidity by adjusting the bound water content, resulting in constant dimensional movement (Menges, 2012). HydroSkin Pavilion, by the Institute for Computational Design (ICD), is a research work into autonomously responsive architectural systems through the use of wood as meteorosensitive material using its hygroscopic properties (Reichert et al., 2014). Conifer cones (Fig. 4) have repetitive opening and closing cycles as responsiveness to humidity changes, and thus, they are biological examples of humidity reactive systems with responsive capacity in the structure of the material itself.

\subsubsection{Temperature reactive materials}

Thermo-Bimetal: sheets of differing metal alloys laminated together. When two metals which, when heated, expand at different rates and they are joined together, the structure that they form will bend as the metals fight each other into contortions and these could provide a useful embedded structural response (Howes \& Laughlin, 2012). Bloom installation is a research project, by Doris Sung about the use of these materials as cladding for buildings, with automatical ventilation based on the difference in temperature between the interior and exterior (Kim Sung, 2013).

Shape memory alloys: smart metals capable of recalling their original shapes. Once deformed, heating them above their transformation temperature will trigger them to return to their original shape. There are a few different varieties, from metals that only remember one shape to metals capable of remembering two shapes, triggered by different temperatures (Howes \& Laughlin, 2012).

Shape memory polymers: smart polymers capable to return from a deformed state, temporary shape, to their original, or permanent shape, induced by an external stimulus, in this case a temperature change.

Thermochromic polymers, inks or glasses: thermochromism is the property of substances to change colour due to a change in temperature. These materials alter their colour in reaction to temperature changes.

Heat sensitive plastics: plastics capable to expand induced by thermal changes (Fig. 5). Combinations of two plastics of differing coefficients of thermal expansion involve heat sensitive actuation. Other 


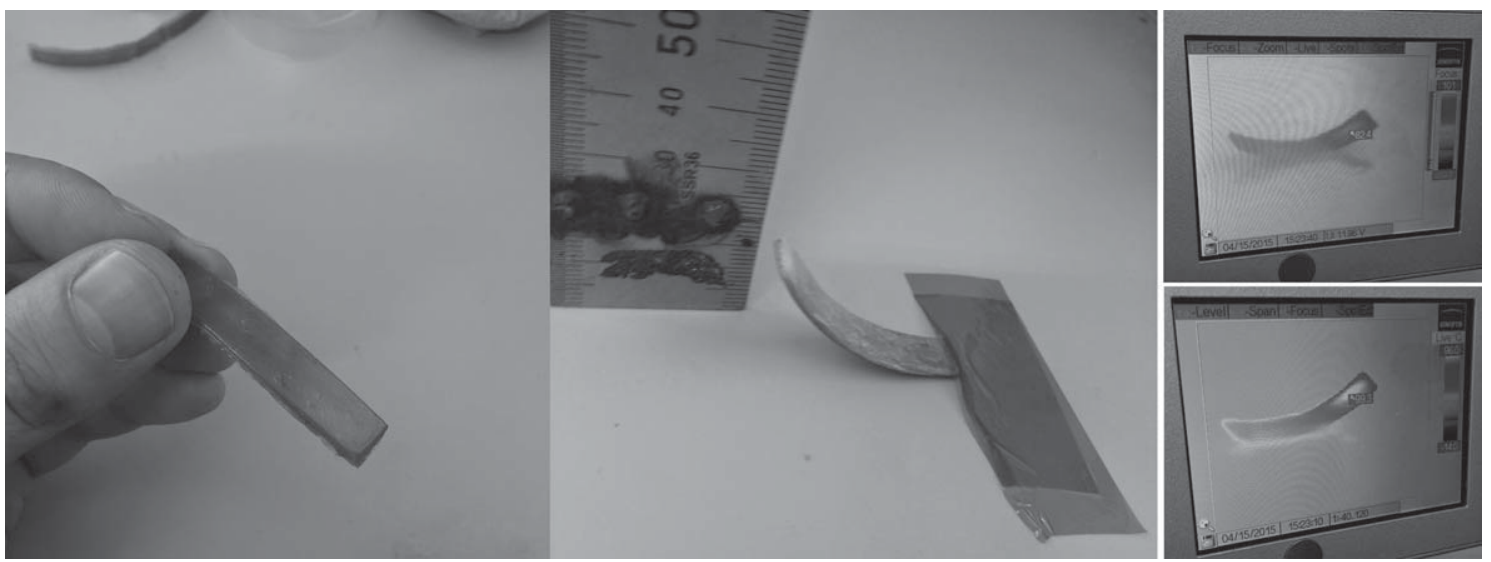

Fig. 5. Experiments of heat sensitive plastic in the UCL Healthcare Biomagnetics Laboratories (The Royal Institution of Great Britain) where an infrared camera is used to capture the heat distribution triggered through the temperature changes.

polymers can also be blended to enhance this process such as Ultra High Molecular Weight Polyethylene (UHMWPE), which expands a lot under heating but is hard to process on its own. Microfluidic channels and high surface area structures could also be added to enhance or decrease actuation effects in a smart design.

\subsubsection{Carbon dioxide reactive materials}

$\mathrm{CO}_{2}$ responsive polymers: responsive polymers use carbon dioxide as a green or eco-trigger as well as to absorb $\mathrm{CO}_{2}$ directly from air. Two main types of materials are distinguished: carbon dioxide responsive polymers and carbon dioxide polymers for $\mathrm{CO}_{2}$ capture (Lin \& Theato, 2013).

\subsubsection{Light reactive materials}

Photochromic dyes: photochromism is a reversible change of colour upon exposure to UV light. Under the influence of UV light, a photochromic molecule will change shape, opening up into a very effective absorber of visible light form. This colour change is a reversible equilibrium, when the source of radiation is removed the molecule will revert back to its inactivated or 'resting' state.

Light responsive polymers: functional polymers that are able to undergo light-induced shape changes. Polymer systems that convert photo-induced effects at the molecular level to macroscopic movement of working pieces, such as contraction and bending of azobenzene-containing liquid-crystal elastomers and volume changes in gels. Some advanced materials researches focus on light-induced shape-memory polymers that can be deformed and temporarily fixed in a new shape (Jiang et al., 2006). After this first approach according to requirements of the environmental issues, a second requirement to select materials would be manufacturing tools. In last years, the huge potential of additive manufacturing and three-dimension printing technologies to generate active systems and structures with complex geometry, have been tested. Printing technologies have developed at an exceptional accuracy, speed, material property and manufacturing cost (Raviv et al., 2014). Recently, some research groups around the world, have gone one step further and have started to work around four-dimension printing concept, which allows materials to self-assemble into 3D structures, adding the extra dimension of time. Some teams, such as those made up by Skylar Tibbits or H. Jerry Qi and Martin L. Dunn, develop and test methods for 4D printing, by means of smart materials to create 

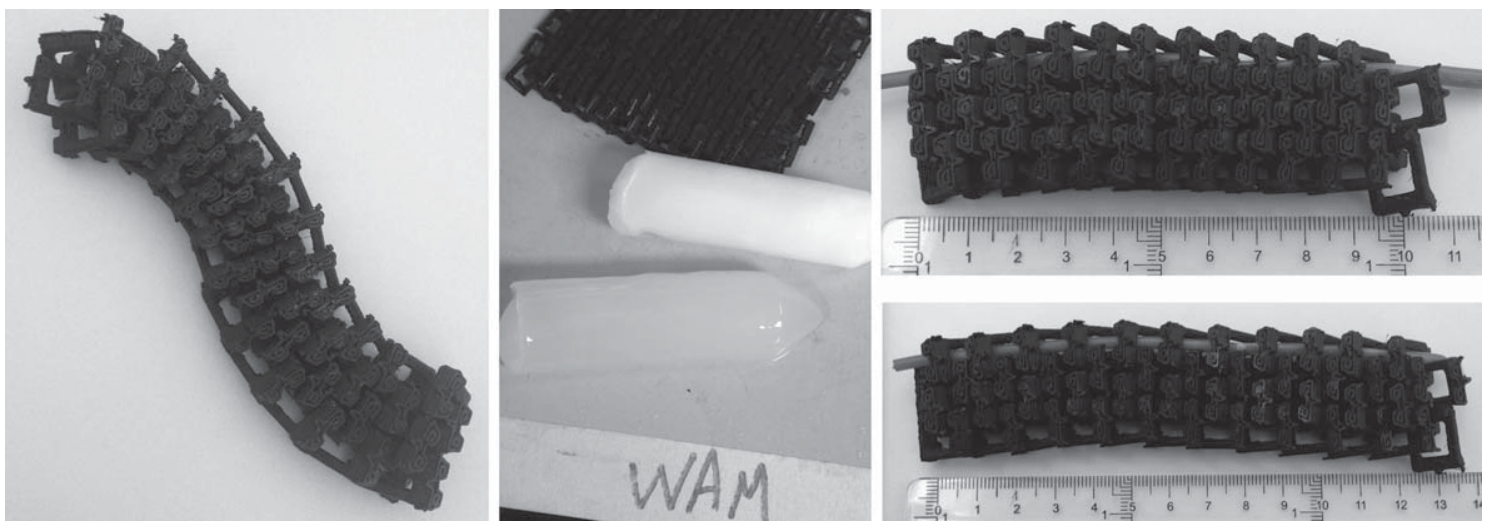

Fig. 6. Experiments on $3 D$ printing technologies, from left to right: $3 D$ printed flexible chain mail, it has no motion for itself; possible joining combinations of chain mail with other sensitive materials; theoretical opening or closure of chain mail triggered through the temperature changes of sensitive plastic, it is a reactive structure with motion for itself.

structures that can assemble themselves. Active fibers can be incorporated into composite materials so their behaviour can be predictably controlled when the object is subjected to thermal and mechanical forces (Ge et al., 2013; Ryu et al., 2012; Raviv et al., 2014). This 4D technology provides a new approach to creating reversible 3D surfaces and promises exciting new possibilities, among others the technical implementation for adaptive architectural envelopes we are looking into. Consequently, materials with special properties to be manipulated and used in additive manufacturing will be selected (Fig. 6).

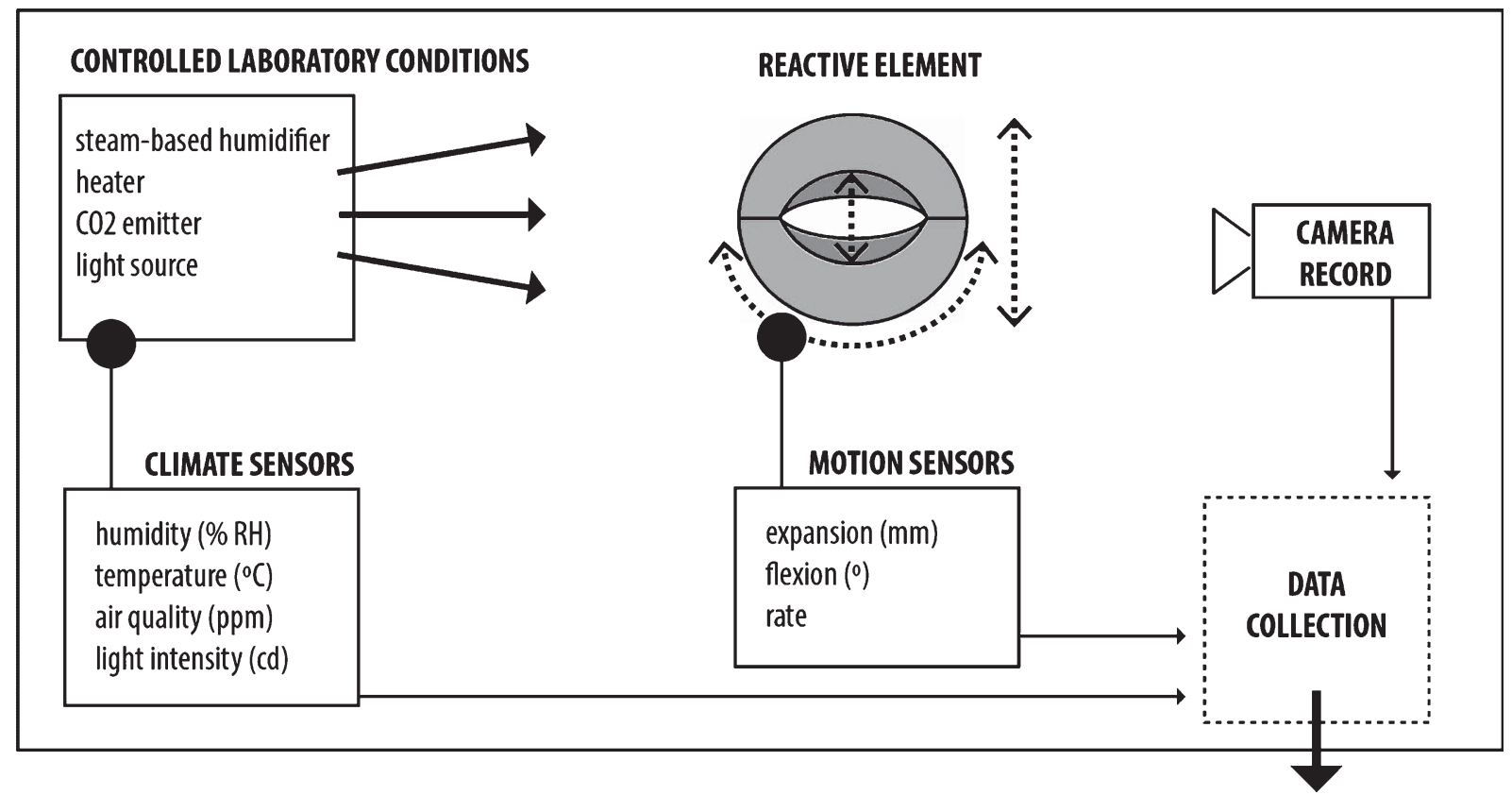

Fig. 7. Scheme idea of the testing scenario, a homogeneous climate chamber with controlled laboratory conditions to verify different motions. 
Several experiments, with single active materials in simple geometries by $3 \mathrm{D}$ printing, will be carried out in order to elaborate laboratory tests in a climate-box (Fig. 7). Using the same shape element as a starting point, we will control the values of the environmental parameters to observe the response range and behaviour of different materials. These tests should show dimensional changes on element surface (i.e. opening, closing, expansion, fold and other transformations) caused by varying environmental issues, and thus, demonstrate the material capacity to rapidly respond to changes in temperature, humidity, carbon dioxide or light. Through these experiments we try to explore the interrelationships between active materials research and its response to external stimuli (humidity, temperature, carbon dioxide and light) seeking meaningful ways to bridge between the natural inspiration, stoma, and the technical implementation, adaptive architectural envelope.

Once these individual experiments have been made and tested, the next step towards the final prototypes is the union of different materials with different features to manage hybrid materials, that is materials or active composites which can embody multiple functionalities. The materials needed for fabrication of humidity reactive systems may be different from the materials used in temperature reactive mechanisms. Therefore, techniques of multi-material printing are needed to be embedded into a single 3D system or structure to control several environmental issues simultaneously, and thus implemented in the multi-adaptive architectural envelope.

\section{Conclusions}

The research focuses on the relationship that can be developed between active materials and environmental issues in order to propose innovative and low-tech design strategies to achieve living envelopes according to stomatal movements in plants, as a result of the application of biomimetics in architecture. Environmental issues selected are humidity, temperature, carbon dioxide and light and we try to select active materials that respond to changes in these elements. Some of these active materials include: heat sensitive plastics, wood or carbon dioxide responsive polymers, with capabilities such as programmable actuation, sensing, self-transformation and workable in 3D printer technologies. The next step in the research is the development of several experiments with single active materials in simple geometries by $3 \mathrm{D}$ printing to elaborate laboratory tests in a climate-box in order to demonstrate dimensional changes on surface elements caused by varying environmental issues; to carry out experiments in active material elements with behaviours as actuators for innovative and low-tech design strategies, and subsequently to achieve hybrid materials or active composites which can embody multiple functionalities.

\section{Acknowledgments}

The authors are grateful for financial support of their work by the nominative subsidy to the University of Oviedo for R\&D projects of the 'Conserjeria de Economia y Empleo del Principado de Asturias' (ref. FC-15-GRUPIN14-004).

\section{References}


Almusaed, A., (2011). Biophilic and Bioclimatic Architecture. Analytical Therapy for the Next Generation of Passive Sustainable Architecture. London: United Kingdom: Springer-Verlag.

Armstrong, R. (2012). Living Architecture. How Synthetic Biology Can Remake Our Cities and Reshape Our Lives. Kindle Single, TED Books. [online]. Available at: http://www.amazon.co.uk/Living-Architecture-Synthetic-Biology-ebook/dp/B0076QQJMY

Azcón-Bieto, J. \& Talón, M. (2000). Fundamentos de fisiología vegetal. Barcelona, Spain: McGraw-Hill Interamericana.

Badarnah, L. (2012). Towards the LIVING envelope: Biomimetics for building envelope adaptation. Delft University of Technology. doi:10.4233/uuid:4128b611-9b48-4c8d-b52f-38a59ad5de65

Beesley, P. (2006). Responsive Architectures: Subtle Technologies. Canada: Riverside Architectural Press.

Bhushan, B. (2009). Biomimetics: Lessons from nature - an overview. Philosophical Transactions of the Royal Society, 367, 1445-1486. doi: 10.1098/rsta.2009.0011

Del Grosso A. E. \& Basso P. (2010). Adaptive building skin structures. Smart Materials and Structures, 19, 12. doi:10.1088/0964-1726/ $19 / 12 / 124011$

Dobzhansky, T., Hecht, M. K., Steere, W. C. (1968). On some fundamental concepts of evolutionary biology. Evolutionary Biology, 2, 1-34. New York, NY: Appleton-Century-Crofts.

Fortmeyer, R. \& Linn, C. (2014). Kinetic Architecture: Design for Active Envelopes. Mulgrave, Australia: Images Publishing.

Ge, Q., Qi, H. J., Dunn, M. L. (2013). Active materials by four-dimension printing. Applied Physics Letters, 103(13): 131901. doi: 10.1063/ 1.4819837

Gibson, L. (2012). The hierarchical structure and mechanics of plant materials. Journal of the Royal Society Interface, 12, 106. doi: 10.1098/ rsif.2012.0341

Howes, P., Laughlin, Z. (2012). Material Matters: New Materials in design. London, United Kingdom: Black Dog Publishing.

Jiang, H. Y., Kelch, S., Lendlein, A. (2006). Polymers move in response to light. Advanced Materials, 18(11), 1471-1475. doi: 10.1002/ adma.200502266

Kamal-Chaoui, L. \& Robert A. (2009). Competitive Cities and Climate Change. OECD Regional Development Working Paper. Paris, France: OECD.

Kim Sung, D. (2013). Metal that breathes, On-line: TEDxUSC. Available at: http://www.ted.com/speakers/doris_kim_sung.html

Koch, K., Bhushan, B., and Barthlott, W. (2009). Multifunctional surface structures of plants. An inspiration for biomimetics. Philosophical Transactions of the Royal Society, 367, 1487-1509. doi: 10.1098/rsta.2009.0022

Lin, S., Theato, P. (2013). $\mathrm{CO}_{2}$-responsive polymers, Macromolecular Rapid Communications, 34(14), 1118-1133. doi: 10.1002/marc. 201300288

Martin, E. S., Donkin, M. E. and Stevens, R. A. (1983). Stomata, London: Edward Arnold.

Mazzoleni, I. (2013). Architecture Follows Nature-Biomimetic Principles for Innovative Design, New York, NY: CRC Press.

Menges, A. (2012). Material Computation. Higher Integration in Morphogenetic Design. Architectural Design, 82, London, United Kingdom: Wiley Academy.

Oxman, N. (2012). Programming Matter. Architectural Design, 8, 88-95. doi: 10.1002/ad.1384

Pawlyn, M. (2011). Biomimicry in Architecture. London, United Kingdom: RIBA Publishing.

Park, J. J., Dave, B. (2014). Bio-inspired Parametric Design of Adaptive Stadium Facades. Australasian Journal of Construction Economics and Building Conference Series, 2, 27-35. doi: http://dx.doi.org/10.5130/ajceb-cs.v2i2.3886

Raviv, D., Wei, Z., McKnelly, C., Papadopoulou, A., Kadambi, A., Shi, B., et al. (2014). Active printed materials for Complex self-evolving deformations. Scientific Reports 4, 7422. doi:10.1038/srep07422

Reichert, S., Menges, A., Correa, D. (2014). Meteorosensitive architecture: Biomimetic building skins based on materially embedded and hygroscopically enabled responsiveness. Computer-Aided Design, 60, 50-69. doi: 10.1016/j.cad.2014.02.010

Rivas-Martínez, S. (2011). Worldwide Bioclimatic Classification SystemGlobal Geobotany, 1(1). doi: 10.5616/gg110001

Ryu, J., D'Amato, M., Cui, X., Long, K. N., Qi, H. J., Dunn, M. L. (2012). Photo-origami. Bending and folding polymers with light, Applied Physics Letters, 100(16), 161908.

Spiller, N., Armstrong, R. (2011). It's a Brand New Morning, Protocell Architecture. Architectural Design, 81. London, United Kingdom: Wiley Academy.

Vincent, J., Bogatyreva O., Bogatyrev N., Bowyer, N., and Pahl, K. (2006). Biomimetics: Its practice and theory. Journal of the Royal Society Interface, 3(9), 471-482. doi:10.1098/rsif.2006.0127

Vogel, S. (2012). The life of a leaf, University of Chicago Press. 